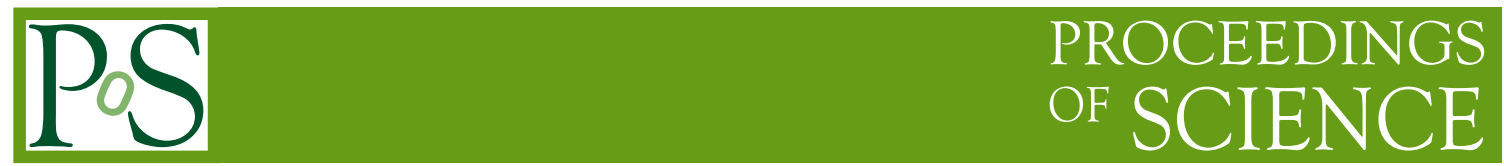

\title{
Supernova Neutrinos in Hyper-Kamiokande
}

\author{
Erin O'Sullivan* \\ On behalf of the Hyper-Kamiokande collaboration \\ Stockholm University \\ E-mail: erin.osullivan@fysik.su.se
}

\begin{abstract}
Hyper-Kamiokande, a next-generation water Cherenkov detector, will be a powerful tool for low energy neutrino astrophysics. Hyper-Kamiokande will begin construction in 2020 with data taking expected in 2027, and will have a fiducial volume of $187 \mathrm{ktons}$. The neutrinos detected from a galactic supernova burst would allow us to probe the detailed inner dynamics of the explosion, as well as give fundamental information about the neutrino itself. Neutrinos that arise from all the supernova explosions in the universe, known as the diffuse supernova neutrino background, will also be measured in Hyper-Kamiokande and will allow us to measure the average properties of supernova explosions.
\end{abstract}

36th International Cosmic Ray Conference -ICRC2019-

July 24th - August 1st, 2019

Madison, WI, U.S.A.

${ }^{*}$ Speaker. 


\section{Introduction}

The Hyper-Kamiokande (Hyper-K) experiment will be uniquely sensitive to neutrinos produced in supernova explosions. Hyper-K will have a large and densely instrumented volume, and will therefore measure supernova neutrinos with high statistics and with detailed event-by-event neutrino energy and timing information. In this proceedings, we outline the capabilities of the experiment to measure neutrinos from the next galactic supernova, the reach of the experiment to measure neutrinos from distant supernovae, and the prospects for measuring the diffuse supernova neutrino background.

\section{Hyper-Kamiokande Detector}

The Hyper-K detector will begin construction in 2020 in the Tochibora Mine site outside of Kamioka, Japan, with data taking expected to begin in 2027. Hyper-K has a variety of physics goals including the measurement of CP-violation in the neutrino sector, searches for nucleon decay, precision measurements of neutrino oscillation parameters, and the detection of astrophysical neutrinos. A complete description of the detector, and more details about the sensitivity of the experiment to various physics studies, can be found in our design report [1].

The detector will have a total (fiducial) volume of 258 (187) kton. The cylindrical tank will be $74 \mathrm{~m}$ in diameter and $60 \mathrm{~m}$ in height, and will be instrumented with 40,000 50cm diameter photomultiplier tubes (PMTs). A schematic of the detector is shown in Figure 1.

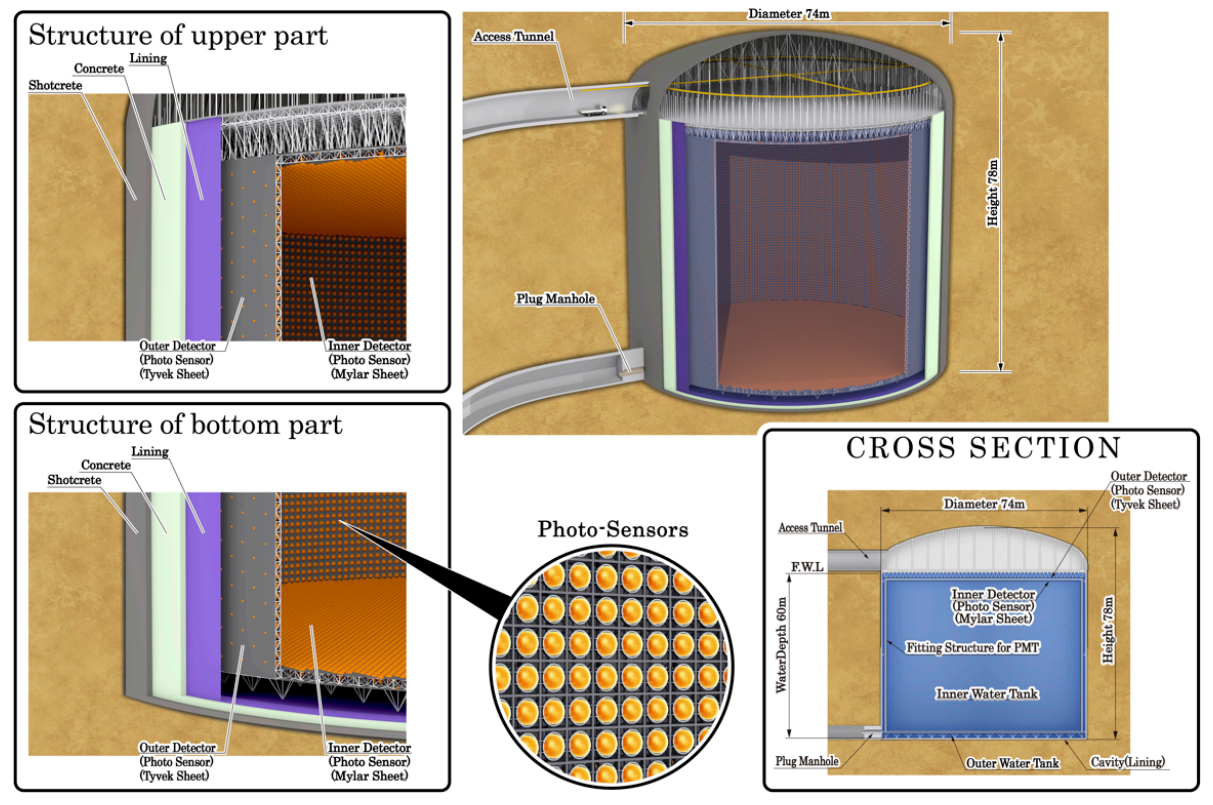

Figure 1: A schematic drawing of the Hyper-Kamiokande detectors.

\section{Detecting a burst of neutrinos from nearby supernovae}

Measuring the rate and energy evolution of neutrinos emitted from a supernova provides a glimpse into the inner workings of these spectacular processes. For a galactic supernova, Hyper-K 
will detect tens of thousands of neutrino events over a short, ten second time window. Figure 2 shows the rate of detected supernova neutrinos as a function of time in HK. Here, we have assumed that we will be able to utilize the full 220 kton inner detector volume as this burst will be short enough in time that the fiducial volume cut we typically apply to analyses to reduce the radioactive backgrounds from the PMT will not be necessary.

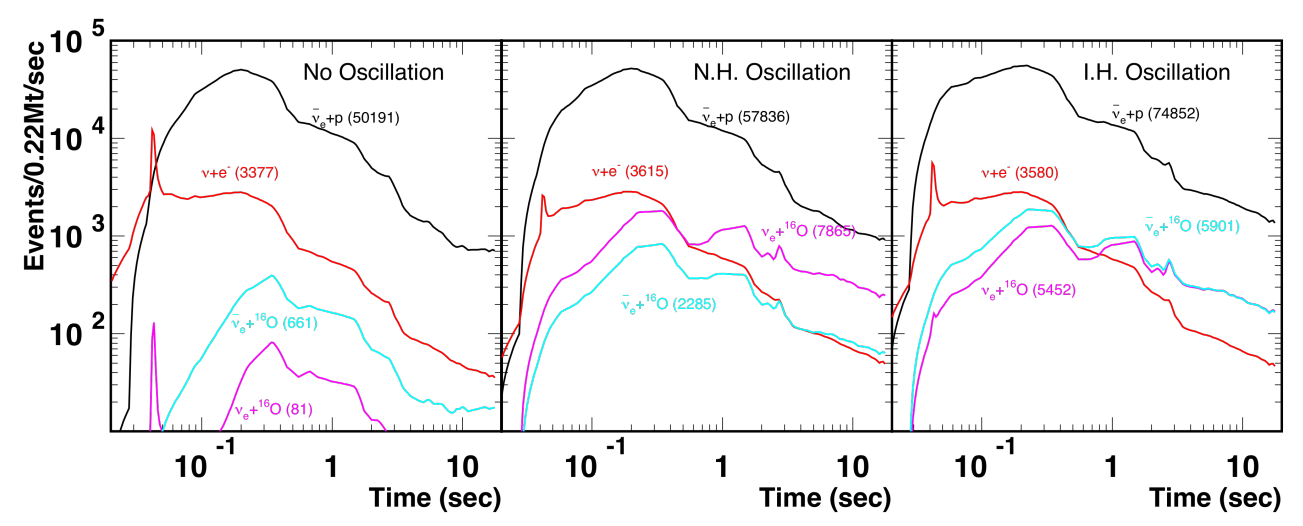

Figure 2: The expected time evolution of the supernova neutrino rate for a $10 \mathrm{kpc}$ supernova using the Livermore model [2]. The three panels depict the case where MSW neutrino mixing is not taken into account (left), the case where neutrinos have normal ordering (middle) and the case where neutrinos have inverted ordering (right). This figure is taken from [1].

Figure 2 also depicts the various interaction channels through which Hyper-K will detect neutrinos. The primary interaction channel is inverse beta decay, where an electron anti-neutrino interacts with a free proton in the water, producing a neutron and a positron. The second more prevalent interaction is elastic scattering, where a neutrino scatters off an electron. This is a particularly important interaction because the detected electron points back in the direction of the incoming neutrino, and can therefore be used as a means to localize the supernova event. Figure 3 shows the expected angular distribution of the detected neutrino events for four different energy slices. The energy dependence here arises from the differing energy profiles of the elastic scattering interaction relative to the dominant inverse beta decay interaction. This energy dependence can be used to further enhance the pointing power of the detected events. For a $10 \mathrm{kpc}$ supernova, and assuming the Livermore model predictions [2], Hyper-K will be able to point with an accuracy of $1-1.3^{\circ}$ depending on the neutrino hierarchy.

\section{Detecting distant supernovae}

Detecting a galactic supernova in Hyper-K will result in detailed information about the mechanism of the explosion. However, only a few supernovae are expected to occur per century in our galaxy. Hyper-K will be able to detect more distant supernovae, and this improved reach will mean that the frequency of detecting a burst of supernova neutrinos will be higher.

Figure 6 shows the probability of detecting a given number of neutrinos from a supernova as a function of distance. Given the low detection multiplicity, we assume here that we are limited to 

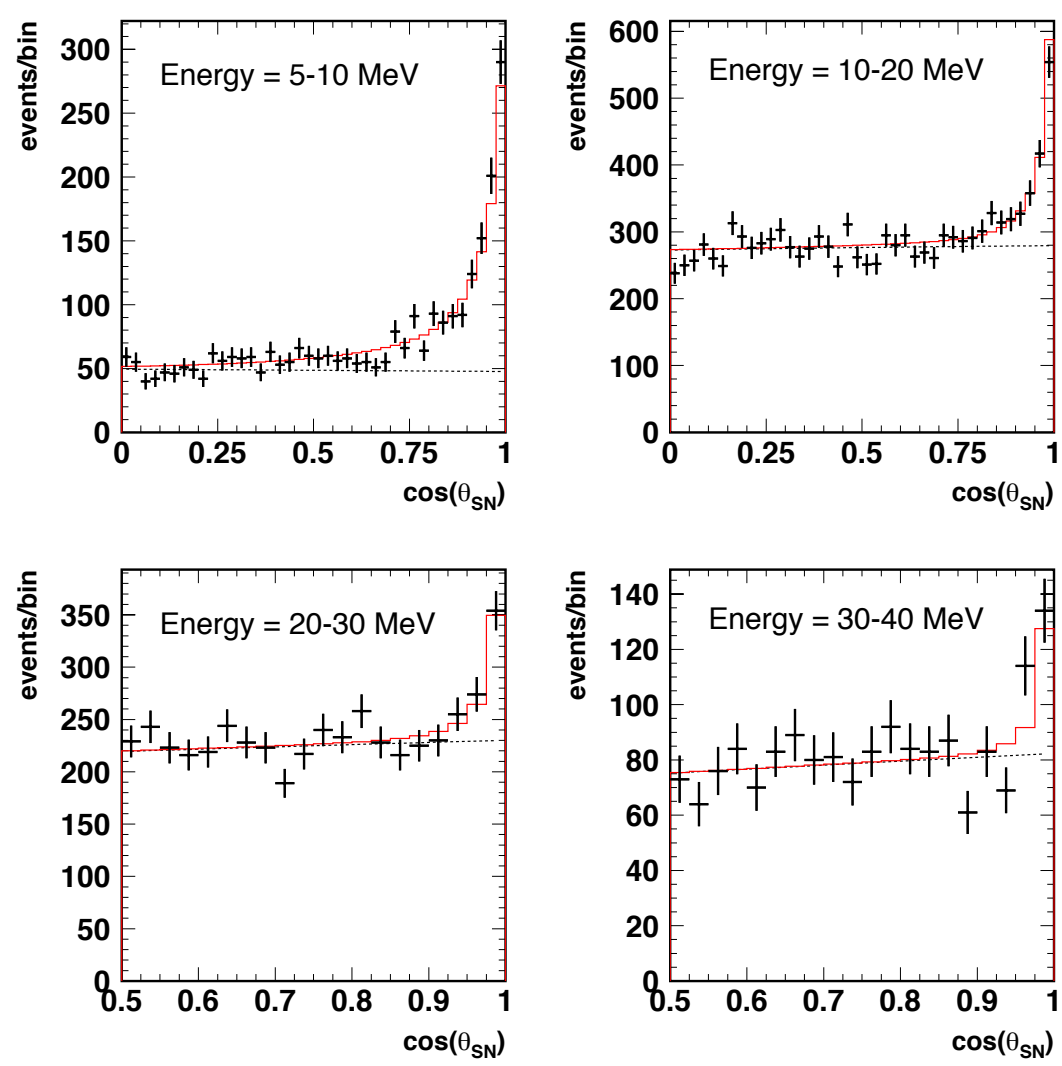

Figure 3: The angular distribution of the detected leptons with respect to the true supernova direction, derived from simulation. This is shown for four different course energy bins, $5-10 \mathrm{MeV}, 10-20 \mathrm{MeV}, 20-30$ $\mathrm{MeV}$, and 30-40 MeV. The black data points represent the simulated data, the red line is the best fit for the elastic scattering channel, and the black dashed line shows the best fit of the inverse beta decay channel. This figure is taken from [1].

using the 187 kton fiducial volume for detection. Figure 5 shows the expected supernova rate as a function of distance.

\section{Detecting the diffuse supernova background}

Measuring a burst of supernova neutrinos allows us to infer information about a specific supernova event, however the rarity of nearby supernovae is a challenge. There is a steady background of neutrinos from distant supernovae, known as the diffuse supernova neutrino background (DSNB). Measuring the DSNB enables us to infer information about the average behaviour of supernovae. The DSNB is not yet measured, though there is the potential for a first detection to be made in the coming years by SK-Gd [4].

Figure 6 shows the anticipated sensitivity of Hyper-K to the DSNB signal. We show here the scenario of having two identical Hyper-K detectors (374 kton fiducial volume), in addition to the baseline design of a single tank (187 kton).

Hyper-K will search for DSNB neutrinos in the energy window between 16 and $30 \mathrm{MeV}$. At energies lower than this, backgrounds from spallation products will dominate, while at higher 


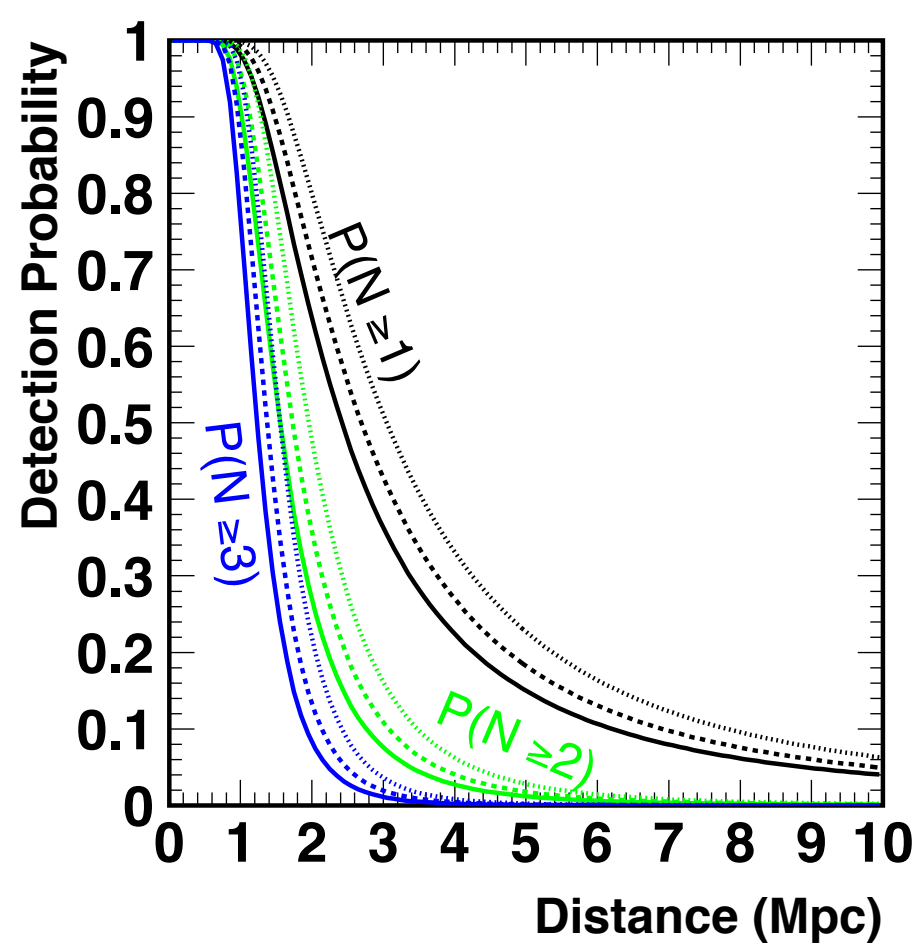

Figure 4: The detection probability for supernova neutrinos as a function of distance. Here we show the requirement that we detect a one or more neutrino (black), two or more neutrinos (green) or three or more neutrinos (blue). This is shown for the no oscillation (solid), normal ordering (dotted), and inverted ordering (dashed) line. This figure was taken from [1].

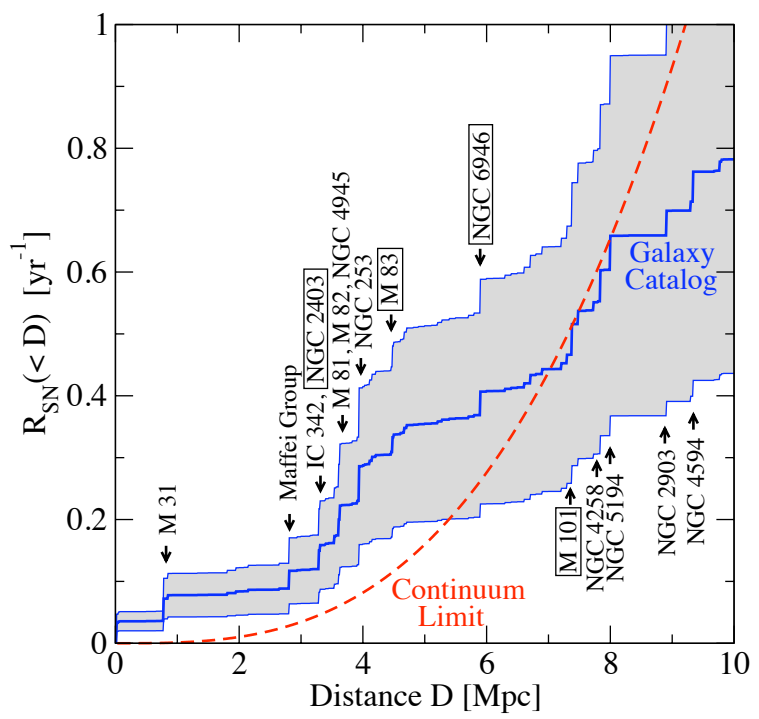

Figure 5: The number of expected supernovae in our local Universe. The dashed line is the estimated rate based on the GALEX $\mathrm{z}=0$ star formation rate. The blue line is the estimate using a galaxy catalog. This figure is reproduced from [3].

energies the background from atmospheric neutrinos becomes significant. This energy range is 


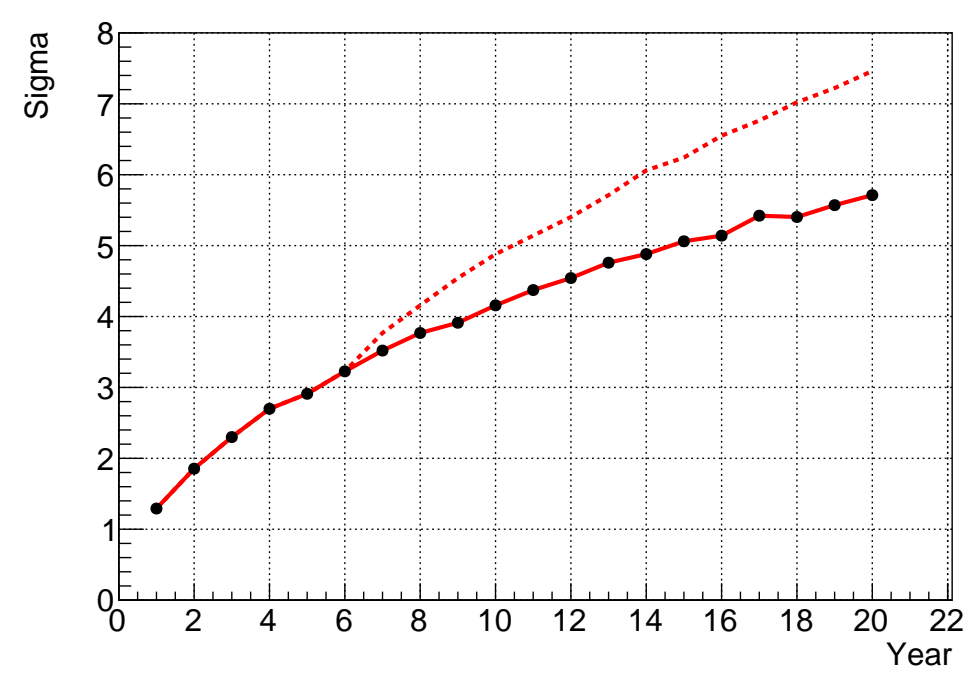

Figure 6: The sensitivity of the Hyper-K experiment to the DSNB for 20 years of experimental livetime. The solid line assumes $187 \mathrm{kton}$ of fiducial volume for the entirety of the experiment. The dashed line assumes a staged scenario, where a second detector comes online 6 years after the first tank. This figure is taken from [1].

higher than that of SK-Gd, which will have a lower energy threshold of $10 \mathrm{MeV}$. Improving the lower bound to $10 \mathrm{MeV}$ in Hyper-K might be possible with the addition of $\mathrm{Gd}$, which would allow us to probe deeper out in redshift (to $\mathrm{z} \sim 1$ ).

\section{Conclusions}

In these proceedings, we have summarized the unique supernova neutrino detection capability of the Hyper-Kamiokande experiment. When Hyper-K begins data taking, it will measure event-byevent information for neutrinos from a galactic supernova. Hyper-K will also have unprecedented reach for distant supernovae from neighboring galaxies, as well as the potential to make a precision measurement of the DSNB.

\section{References}

[1] Hyper-Kamiokande Collaboration, K. Abe et al., arXiv:1805.04163.

[2] T. Totani, K. Sato, H. E. Dalhed, and J. R. Wilson, Astrophys. J. 496 (1998) 216-225.

[3] S. Ando, J. F. Beacom, and H. Yuksel, Phys. Rev. Lett. 95 (2005) 171101.

[4] J. F. Beacom and M. R. Vagins, Phys. Rev. Lett. 93 (2004) 171101. 